\title{
SINGULAR FINITE ELEMENTS FOR THE SUDDEN-EXPANSION AND THE DIE-SWELL PROBLEMS
}

\author{
GEORGIOS C. GEORGIOU \\ Department of Chemical Engineering, The University of Michigan, H. H. Dow Building, Ann Arbor, MI 48109, U.S.A. \\ AND \\ WILLIAM W. SCHULTZ AND LORRAINE G. OLSON \\ Department of Mechanical Engineering and Applied Mechanics, The University of Michigan, Ann Arbor, MI 48109, \\ U.S.A.
}

\begin{abstract}
SUMMARY
The singular finite element method is used to solve the sudden-expansion and the die-swell problems in order to improve the accuracy of the solution in the vicinity of the singularity and to speed up the convergence. The method requires minor modifications to standard finite element schemes, and even coarse meshes give more accurate results than refined ordinary finite element meshes. Improved normal stress results for the sudden-expansion problem have been obtained for various Reynolds numbers up to 100 using the singular elements constructed for the creeping flow problem. In addition, the normal stresses at the walls appear to be insensitive to the singularity powers used in the construction of the singular basis functions. The die-swell problem is solved using the singular elements constructed for the stick-slip problem. The singular elements accelerate the convergence of the free surface dramatically.
\end{abstract}

KEY WORDS Singular finite elements Die swell Sudden expansion

\section{INTRODUCTION}

Stress singularities in fluid mechanics arise whenever there is an abrupt change in a boundary condition or in the boundary itself. Some well known examples of singular problems are the sudden-expansion, the die-swell, the stick-slip and the driven cavity problems, which are often used as model problems for the various numerical methods proposed for Newtonian or nonNewtonian flow.

Singularities require special attention no matter what numerical method is used. The most common treatment is to refine the grid around the singular point in order to capture the abrupt changes in the flow field. However, the rate of convergence and the accuracy are generally unsatisfactory. The stresses cannot be infinite (in compliance with the asymptotic solution) and are tainted by spurious oscillations. This contamination is far more serious in non-Newtonian flows than in the corresponding Newtonian flows. Numerical inaccuracies caused by singularities can lead to numerically stiff iteration schemes, to the formation of fictitious limit points or to artificial changes of type of the governing equations. ${ }^{1,2}$ Another inherent disadvantage in local refinement is the generation of extremely large matrices, resulting in higher computational costs. 
Some investigators have modified the mathematical problem to alleviate the singularity by (a) modifying the boundary conditions (e.g. relaxing the no-slip condition for a smooth transition from a velocity to a stress boundary condition ${ }^{3}$ ) or (b) smoothing the boundary (e.g. replacing a re-entrant corner by a circular arc of small radius ${ }^{1,5}$ ).

An alternative approach, based on the acknowledgement of the singularity rather than on its alleviation, is the incorporation of the local asymptotic solution (if known) into the numerical scheme (finite differences, finite elements, boundary elements, etc.). This idea has been extensively used in fracture mechanics and gives accurate results for relatively coarse meshes. ${ }^{6,7}$ More recently, singular methods were used to solve singular fluid mechanics problems with finite differences, ${ }^{8}$ boundary elements ${ }^{9}$ and finite elements. ${ }^{10,11}$ In the singular finite element method (SFEM), special elements that take into account the nature of the singularity are used in a small core around the singular region and ordinary elements are used in the rest of the domain. The basis functions for the primitive variables over the singular elements embody the radial form of the singularity, which can be obtained by an asymptotic analysis.

A feature of the singular fluid flow problems that does not appear in fracture mechanics is that, in addition to the velocity derivatives, the pressure, a primitive variable, is singular. ${ }^{10}$ An additional complication arises in free surface problems since the angle of separation is unknown and the boundaries are curved; hence the exact form of the singularity is unknown.

The SFEM has been used recently by the authors to solve the Newtonian stick-slip problem. ${ }^{10}$ The results in Reference 10 indicate that the SFEM improves the stress representation and gives more accurate results than those from more refined ordinary finite element meshes. It was also noted that the method can be applied to other singular fluid flow problems for which the radial form of the singularity can be obtained by a local analysis. Such analyses are possible with various geometries for both Newtonian ${ }^{12-14}$ and non-Newtonian ${ }^{4,15-17}$ flows; in some non-Newtonian flows the local solution is identical to that of the Newtonian case. Thus the SFEM is applicable to some non-Newtonian flows provided the encountered stresses are integrable. The SFEM is also suited to non-zero Reynolds number flows, because the local solution remains unchanged near the singularity where the viscous effect dominates. This implies that the singular elements constructed for creeping flows can be used to solve the non-zero Reynolds number problems as well.

With this background in mind, the main objective of this paper is to generalize the SFEM for flows with different singularity powers, non-zero Reynolds number flows and flows with free surfaces. We chose to solve two singular problems: (a) the planar 2:1 sudden-expansion problem and (b) the planar die-swell problem. These problems are important in polymer processing and in other industrial applications and have been the subject of a considerable amount of experimental and numerical work (e.g. References 18 and 19). Even though our work aims towards solving viscoelastic flows, which are more challenging than their Newtonian counterparts, here we restrict ourselves to the Newtonian cases. Despite the simplification introduced by the Newtonian assumption, the two problems are analytically intractable owing to the singularities and the nonlinearity of the convective terms. In the case of the die-swell problem, the boundary conditions on the free surface (the location of which is unknown) are also non-linear. Numerical methods, especially finite elements, have been used extensively to overcome all these difficulties. The free surface location is computed by either Picard iteration ${ }^{3,19.20}$ or full Newton iteration schemes. ${ }^{21,22}$

The sudden-expansion problem was solved for different Reynolds numbers up to 100 using the singular elements constructed for creeping flow. The SFEM performs well for non-zero Reynolds numbers and yields more accurate predictions than the ordinary finite element method. The solution also appears to be rather insensitive to substantial variations of the powers used in the 
construction of the basis functions. It seems that the elimination of the pressure node from the singular point plays a crucial role in the improvement of the solution.

The planar die-swell problem was solved by singular finite elements, free surface parameterization and full Newton iteration. The singular elements developed for the stick-slip problem were used for the die-swell problem over the full range of Reynolds and capillary numbers examined. As we will see in the following sections, the SFEM speeds up the convergence of the free surface dramatically.

The governing equations and the local solutions around the singularities are presented in Section 2, the construction of the singular basis functions and the finite element formulation are presented in Section 3 and the results are discussed in Section 4.

\section{GOVERNING EQUATIONS}

The flow geometry, the governing equations and the boundary conditions for the suddenexpansion and the die-swell problems are depicted in Figures 1(a) and 2(a) respectively. The flows are governed by the two-dimensional momentum equation and continuity; for incompressible flow and neglecting gravity,

$$
\operatorname{Re} \mathbf{u} \cdot \nabla \mathbf{u}=\nabla \cdot \mathbf{T}
$$

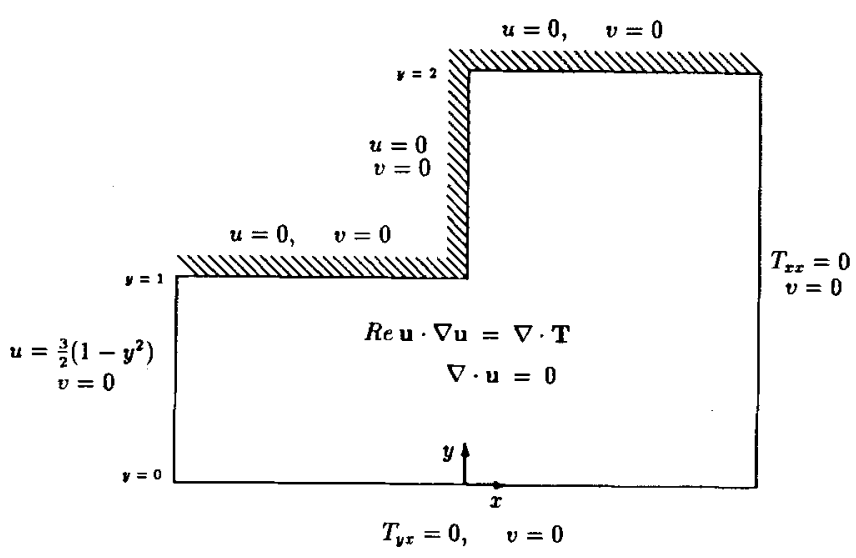

(a)

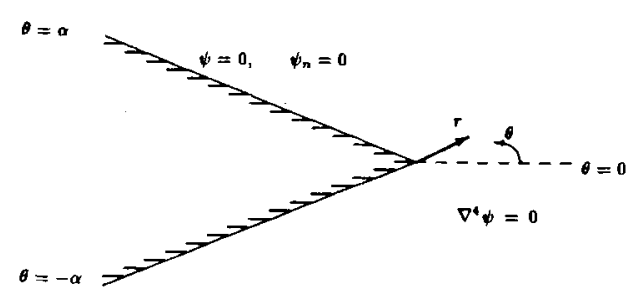

(b)

Figure 1. (a) Sudden-expansion problem. (b) Local analysis of the singularity 


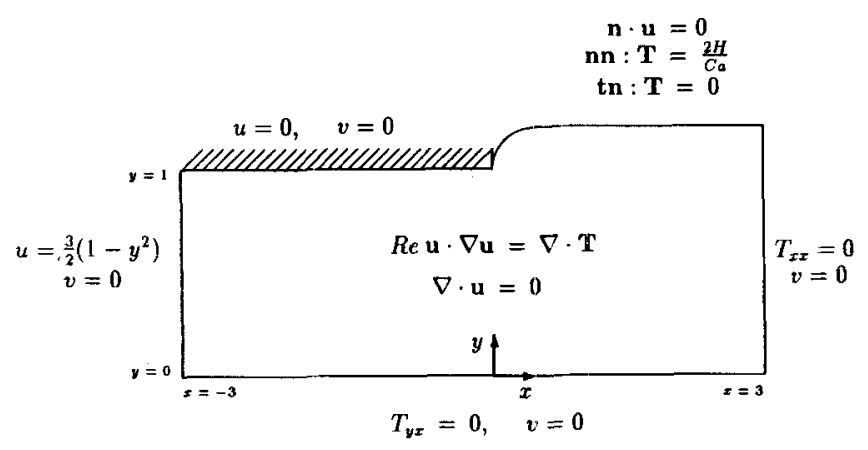

(a)

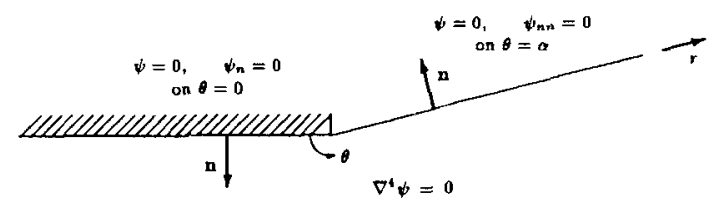

(b)

Figure 2. (a) Die-swell problem. (b) Local analysis of the singularity

and

$$
\nabla \cdot \mathbf{u}=0,
$$

where length is measured in units of the entrance half-width $H$, the velocity $\mathbf{u}$ is scaled by the mean velocity $U$ in the entrance channel, the Newtonian stress tensor $\mathbf{T}=-p \mathbf{I}+\nabla \mathbf{u}+(\nabla \mathbf{u})^{\mathbf{T}}$ is scaled by $\mu U / H$, and $\mu$ is the viscosity. The Reynolds number is defined as

$$
R e \equiv \frac{\rho U H}{\mu},
$$

where $\rho$ is the density.

In addition to the two velocity components $u$ and $v$ and the pressure $p$, the free surface location $h$ is an additional unknown in the die-swell problem. The kinematic condition that the free surface remain a material surface provides the additional equation needed:

$$
\mathbf{n} \cdot \mathbf{u}=0,
$$

where $\mathbf{n}$ is the unit normal vector pointing outwards from the free surface.

The remaining two conditions on the free surface serve as the boundary conditions in our formulation. A momentum balance on the free surface requires the shear stress to vanish and the normal stress in the liquid to balance any capillary pressure, or

$$
\mathbf{n} \cdot \mathbf{T}=\frac{2 H}{C a} \mathbf{n},
$$


where $C a$ is the capillary number,

$$
C a \equiv \frac{\mu U}{\sigma},
$$

$\sigma$ is the surface tension and $2 H$ is the mean curvature of the free surface, given by

$$
2 H=\frac{h_{x x}}{\left(1+h_{x}^{2}\right)^{3 / 2}} .
$$

\subsection{Local analysis of the singularity}

For the local analysis of the singularity we follow Michael ${ }^{12}$ and Moffatt ${ }^{13}$ and switch to planar polar co-ordinates $(r, \theta)$ (Figures $1(b)$ and 2(b)). Using a streamfunction representation, the Stokes equation admits separated solutions of the form

$$
\psi=r^{\lambda} f_{\lambda}(\theta),
$$

where $\psi$ is the streamfunction, $\lambda$ are the eigenvalues of the resulting eigenproblem and $f_{\lambda}(\theta)$ is given by the general expression

$$
f_{\hat{\lambda}}(\theta)=A \cos \lambda \theta+B \sin \lambda \theta+C \cos (\lambda-2) \theta+D \sin (\lambda-2) \theta .
$$

$A, B, C$ and $D$ are arbitrary constants determined from the boundary conditions. We require that $\operatorname{Re}(\lambda)>1$ to ensure that the velocity goes to zero at the singular point. For flow between two rigid walls meeting at a sharp corner, as in Figure 1(b), we have two sets of solutions that correspond to the antisymmetrical and the symmetrical flows. For the antisymmetrical flow,

$$
\psi=a_{\lambda} r^{\lambda}[\cos (\lambda-2) \alpha \cos \lambda \theta-\cos \lambda \alpha \cos (\lambda-2) \theta],
$$

where $\alpha$ is the angle defined in Figure 1(b), $a_{\lambda}$ is a constant and $\lambda$ satisfies the equation

$$
\sin 2 \mu \alpha=-\mu \sin 2 \alpha \text { with } \mu=\lambda-1 \text {. }
$$

For the symmetric case,

$$
\psi=b_{\lambda} r^{\lambda}[\sin (\lambda-2) \alpha \sin \lambda \theta-\sin \lambda \alpha \sin (\lambda-2) \theta],
$$

where $b_{\lambda}$ is a constant and $\lambda$ satisfies

$$
\sin 2 \mu \alpha=\mu \sin 2 \alpha \quad \text { with } \mu=\lambda-1 .
$$

For the sudden-expansion problem $\alpha$ is $3 \pi / 4$; the first eigenvalues from equations (11) and (13), ordered by increasing real part, are listed in Table I. It is worthwhile to note the following:

Table I. Leading eigenvalues $\mu=\lambda-1$ of the two asymptotic solution sets (sudden expansion)

\begin{tabular}{lll}
\hline No. & Antisymmetrical & Symmetrical \\
\hline 1 & $0 \cdot 54448$ & $0 \cdot 90853$ \\
2 & $1 \cdot 62926 \pm 0 \cdot 23125 \mathrm{i}$ & $2 \cdot 30133 \pm 0 \cdot 31584 \mathrm{i}$ \\
3 & $2 \cdot 97184 \pm 0 \cdot 37393 \mathrm{i}$ & $3.64142 \pm 0 \cdot 41879 \mathrm{i}$ \\
4 & $4 \cdot 31038 \pm 0 \cdot 45549 \mathrm{i}$ & $4.97890 \pm 0.48663 \mathrm{i}$ \\
5 & $5 \cdot 64711 \pm 0 \cdot 51368 \mathrm{i}$ & $6 \cdot 31508 \pm 0.53763 \mathrm{i}$ \\
6 & $6 \cdot 98287 \pm 0 \cdot 55911 \mathrm{i}$ & $7 \cdot 65051 \pm 0.57859 \mathrm{i}$ \\
7 & $8 \cdot 31803 \pm 0 \cdot 59642 \mathrm{i}$ & $8.98546 \pm 0.61285 \mathrm{i}$ \\
\hline
\end{tabular}


(a) although $\mu=1$ is a solution to (11), it is not an eigenvalue for this problem; ${ }^{14}$ (b) there is an infinite number of eigenvalues for both sets of solutions; (c) there is only one real eigenvalue for each set of solutions and it is less than unity; (d) the real part of the complex eigenvalues is always greater than unity. Holstein and Paddon ${ }^{8}$ observed that the above solution is the zero-order approximation to inertial corner flow and that the Stokesian and inertial corner flows share the first three expansion terms.

An interesting characteristic of the local asymptotic solution of the sudden-expansion problem is that both the antisymmetrical and symmetrical solutions contribute one singular term that causes the stresses to become infinite such as

$$
T_{n n} \sim c_{1} r^{-0.456}+c_{2} r^{-0.091} .
$$

Unlike the stick-slip problem, ${ }^{23}$ the normal stresses along the walls are singular. Along the bisector $(\theta=0)$, the leading-order symmetrical contributions to the radial velocity component and the pressure vanish; the antisymmetrical contributions to the circumferential velocity component also vanish. Therefore, at $\theta=0$ and close to the singular point, the radial velocity component is proportional to $r^{0.544}$ and the circumferential velocity to $r^{0.909}$. The antisymmetrical contribution to the stress also vanishes at $\theta=0$ and the stress is determined by the less singular symmetrical contribution.

For the die-swell problem we consider the flow between a rigid boundary and a free surface as in Figure 2(b). Michael ${ }^{12}$ showed that for zero surface tension on a planar free surface the angle $\alpha$ must be equal to $\pi$. Sturges ${ }^{14}$ noted that this result does not apply to the die-swell problem because the free surface is not planar. Schultz and Gervasio ${ }^{24}$ suggested that either the slope is zero or the mean curvature is infinite. The resulting eigenvalue problem for the geometry in Figure 2(b) is identical to (13); the first two roots for various angles up to $3 \pi / 2$ are listed in Reference 14. However, in the present work we assume that the radial form of the local solution is not very different from the solution for $\alpha=\pi$; therefore the local solution is the same as that of the stick-slip problem:

$$
\psi=r^{\lambda+1} a_{\lambda}[\cos (\lambda+1) \theta-\cos (\lambda-1) \theta] \text { for } \lambda=\frac{1}{2}, \frac{3}{2}, \frac{5}{2}, \ldots
$$

and

$$
\psi=r^{\lambda+1} b_{\lambda}[(\lambda-1) \sin (\lambda+1) \theta-(\lambda+1) \sin (\lambda-1) \theta] \text { for } \lambda=2,3,4, \ldots
$$

Using the same local solution we avoid using different singular functions at different (apparent) angles of separation and finding the angle of separation itself. Note that the singular elements require only a knowledge of the radial form of the local solution. The validity of the above assumption is tested by studying the sensitivity of the results to variations of the singularity powers used in the construction of the basis functions.

\section{FINITE ELEMENT FORMULATION}

The domain is discretized using triangular singular elements around the singular point and rectangular ordinary elements elsewhere, as illustrated in Figures 3 and 4. For the ordinary elements we use biquadratic basis functions for the velocities and bilinear for the pressure. These elements are mapped onto a $2 \times 2$ master element in $(\xi, \eta)$ co-ordinates by means of biquadratic shape functions. As in Reference 10 , the singular elements are collapsed quadrilaterals with 13 velocity and eight pressure nodes mapped onto a $2 \times 215$-node element in the computational domain $(\xi, \eta)$ by means of ordinary fourth-order polynomial shape functions in the $\xi$-direction and second-order in the $\eta$-direction. It is important to stress the following: 


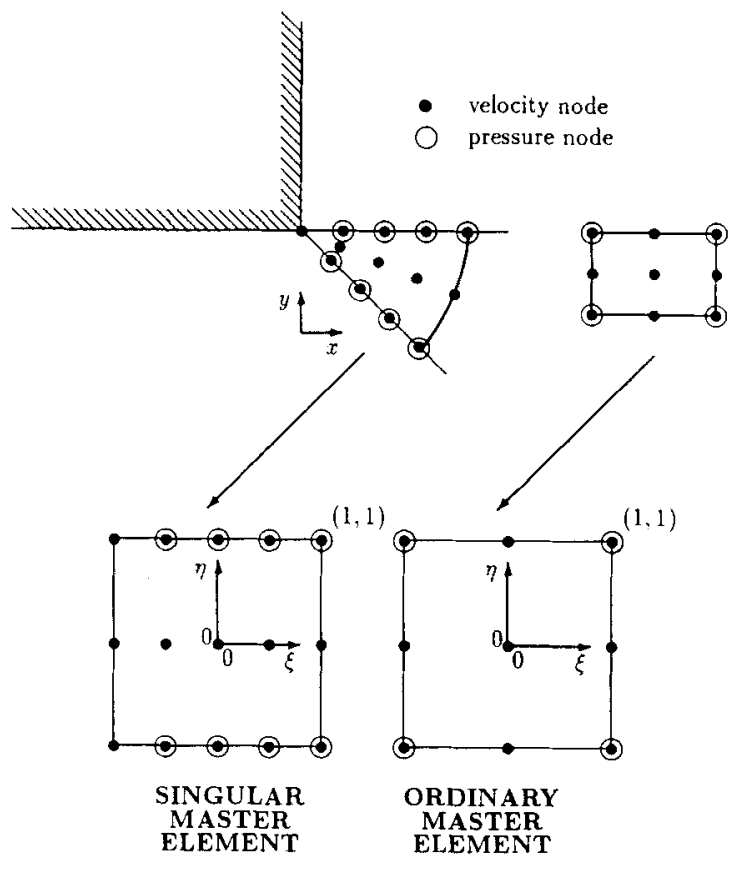

Figure 3. Ordinary and singular elements
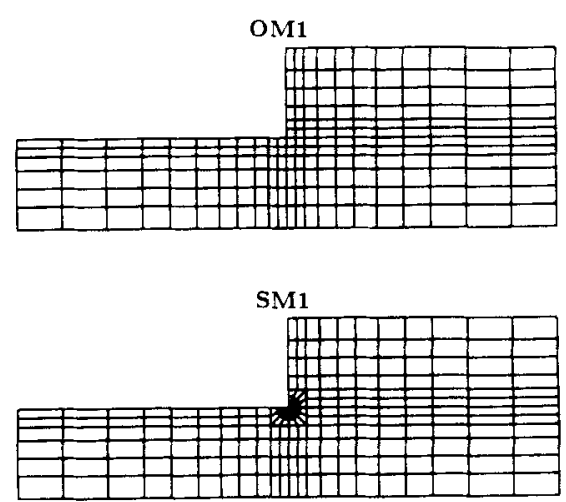

(a)

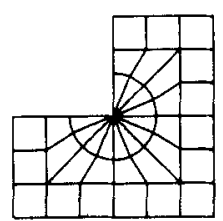

(b)

Figure 4. (a) Parts of the coarsest ordinary and singular meshes for the sudden-expansion problem. (b) Structure of singular meshes near the corner 
(a) The singular elements are compatible with the adjacent ordinary elements at $\xi=1$.

(b) There is no pressure node at the singular point.

(c) The three velocity nodes of the singular master element collapse to a single node with two degrees of freedom at the singular point in the physical domain.

The trial functions for the velocities are of the general form

$$
\Phi^{i}=N^{i}(\xi) P^{i}(\eta),
$$

with $P$ varying quadratically with $\eta$ to maintain compatibility with the adjoining elements. With five nodes in the radial direction $\xi, N$ is given by

$$
N=A_{0}+A_{1} \xi^{n_{1}}+A_{2} \xi^{n_{2}}+A_{3} \xi^{n_{3}}+A_{4} \xi^{n_{4}} .
$$

The constants $A_{i}$ are determined by requiring that $N^{i}\left(\xi_{j}\right)=\delta_{i j}$, where $\xi_{j}$ are the equally spaced positions of the velocity nodes. An alternative way to generate the functions $N^{i}$ is given by Hughes and Akin, ${ }^{25}$ but it is too tedious for the 13-node elements used here. The pressure basis functions are given by lower-order interpolation

$$
\Psi^{i}=M^{i}(\xi) Q^{i}(\eta)
$$

with $Q$ varying linearly with $\eta$ (two pressure nodes in the $\eta$-direction) and $M$ having the same form as $\mathrm{d} N / \mathrm{d} \xi$ :

$$
M=B_{1} \xi^{n_{1}-1}+B_{2} \xi^{n_{2}-1}+B_{3} \xi^{n_{3}-1}+B_{4} \xi^{n_{4}-1} .
$$

Again, the constants $B_{i}$ are determined by requiring that $\Psi^{i}\left(\xi_{j}\right)=\delta_{i j}$, where now $\xi_{j}$ are the positions of the pressure nodes.

For the sudden-expansion problem we require that two terms of the velocity trial functions match the two leading expansion terms and that the other two are linear and quadratic. In other words we choose $n_{1}=0.544, n_{2}=0.909, n_{3}=1$ and $n_{4}=2$. Similarly, for the die-swell problem we take $n_{1}=0.5, n_{2}=1, n_{3}=1.5$ and $n_{4}=2$. Note that for this special case the basis functions are easily obtained using Lagrange interpolation. ${ }^{10}$

Applying Galerkin's principle, we weight the momentum equation by the velocity basis functions $\Phi^{i}$ and apply the divergence theorem,

$$
\int_{S} \frac{2 H}{C a} \mathbf{n} \Phi^{i} d S-\int_{\Omega}\left(\nabla \mathbf{T} \cdot \nabla \Phi^{i}+\operatorname{Reu} \cdot \nabla \mathbf{u} \Phi^{i}\right) \mathrm{d} \Omega=0, \quad i=1,2, \ldots, N_{u},
$$

and we weight continuity by the pressure basis functions $\Psi^{i}$,

$$
\int_{\Omega} \nabla \cdot \mathbf{u} \Psi^{i} \mathrm{~d} \Omega=0, \quad i=1,2, \ldots, N_{p} .
$$

Here $\Omega$ is the domain, $S$ is the free surface boundary (for the die-swell problem), and $N_{u}$ and $N_{p}$ are the numbers of velocity and pressure nodes respectively. In the case of the die-swell problem we also weight the kinematic equation by the free surface quadratic basis functions $W^{i}$ :

$$
\int_{\mathrm{S}} \mathbf{n} \cdot \mathbf{u} W^{i} \mathrm{~d} S=0, \quad i=1,2, \ldots, N_{h},
$$

where $N_{h}$ is the number of free surface nodes. Equations (21)-(23) constitute a non-linear system of equations efficiently solved by the Newton method and standard subroutines, e.g. frontal methods. ${ }^{26}$

The mesh is updated at each iteration by the newly found free surface location values $h_{i}$, which are determined simultaneously with the primary unknowns $u_{i}, v_{i}$ and $p_{i}$. Note that the nodes of the 
singular elements are at a constant distance from the singular point, rotating around the singular point according to the shape and position of the free surface. The nodes of the ordinary elements are fixed at the $x$-co-ordinate and move with the free surface in the $y$-direction so that the relative elevation is constant. The mid-nodes of the transition elements move in both $x$ - and $y$-directions so that they are always at the middle of the elements' sides.

Standard $3 \times 3$ Gaussian quadrature is sufficient for the integration over the ordinary elements. However, the integration over the singular elements requires special or higher-order quadrature rules. ${ }^{10,27}$ The simple quadrature used in Reference 10 for the stick-slip problem is employed for the die-swell problem since the same singular elements are used. Nevertheless, it is tedious to develop a special quadrature for the sudden-expansion problem owing to the many incommensurable powers of the radial co-ordinate that appear in the residual integrands. A higherorder $(10 \times 10)$ Gauss-Legendre integration was used instead.

\section{RESULTS}

\subsection{The sudden-expansion problem}

The 2:1 sudden-expansion problem was solved with both ordinary and singular finite elements for comparison. Results were obtained for various Reynolds numbers ranging from zero to 100 . For our computations we constructed four ordinary (OM1, OM2, OM3 and OM4) and four singular meshes (SM1, SM2, SM3 and SM4). OM2 was obtained from OM1 by refining the columns and rows of elements near the corner. OM3 and OM4 were obtained in a similar manner. Information about the meshes is listed in Table II. The singular meshes were generated from the corresponding ordinary meshes by replacing the 12 rectangular ordinary elements around the singular point with 12 triangular singular elements and 12 quadrilateral transition elements in the circular pattern shown in Figure 4. The radius of the singular elements was taken to be $1 \cdot 2$ times the size of the original ordinary square element.

The meshes were extended to a distance $L_{1}=3$ upstream, a length found adequate to approximate the inlet boundary conditions. As noted in Reference 28, the outlet length $L_{2}$ should be greater than the maximum expected reattachment length plus a section sufficiently long to achieve fully developed flow beyond the point of reattachment. We took $L_{2}=10$ for $\operatorname{Re}$ up to $30, L_{2}=15$ for $R e=50$ and $L_{2}=25$ for $R e=100$, in accordance with the reattachment length estimates in Reference 29 and the outlet lengths used in Reference 28 . No upwinding schemes were used. ${ }^{30}$ We should note here that some of the meshes are very fine for two reasons: (a) to compare results from relatively coarse singular meshes with those from fine

Table II. Data for sudden-expansion meshes $\left(L_{2}=10\right)$

\begin{tabular}{lcccc}
\hline Mesh & $\begin{array}{c}\text { Number } \\
\text { of elements }\end{array}$ & $\begin{array}{c}\text { Number } \\
\text { of nodes }\end{array}$ & $\begin{array}{c}\text { Degrees } \\
\text { of freedom }\end{array}$ & $\begin{array}{c}\text { Size of } \\
\text { corner elements }\end{array}$ \\
\hline OM1 & 294 & 1261 & 2859 & $0 \cdot 100$ \\
OM2 & 364 & 1549 & 3509 & $0 \cdot 050$ \\
OM3 & 440 & 1861 & 4213 & $0 \cdot 020$ \\
OM4 & 522 & 2197 & 4971 & $0 \cdot 010$ \\
SM1 & 306 & 1347 & 3075 & $0 \cdot 130$ \\
SM2 & 376 & 1635 & 3725 & $0 \cdot 065$ \\
SM3 & 452 & 1947 & 4429 & $0 \cdot 026$ \\
SM4 & 534 & 2283 & 5187 & $0 \cdot 013$ \\
\hline
\end{tabular}


ordinary element meshes and (b) to check whether or not very small singular elements are required, given that the form of the singularity is only valid very close to the wall at non-zero $R e$.

In general, the results from both the ordinary and the singular finite elements are in good agreement except in the vicinity of the singular point. In Figure 5 we plot the velocity, pressure and streamline contours at zero Reynolds number predicted with the singular finite elements (mesh SM1).

As expected, the results close to the singular point differ greatly from the ordinary finite element predictions. To illustrate this we computed the normal stresses along the two walls forming the corner. In Figure 6 we compare the normal stresses along the horizontal wall $(y=1)$ and in Figure 7 the normal stresses along the vertical wall $(x=0)$ from meshes OM2, OM4 and SM1. The ordinary element results are characterized by spurious oscillations. The SFEM stresses are

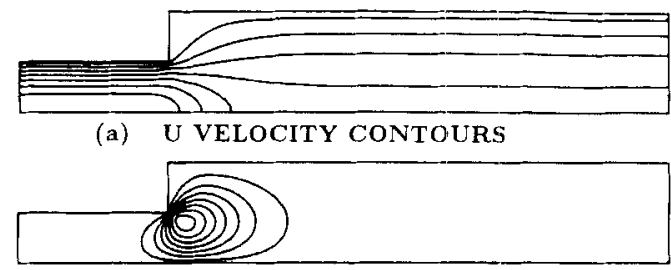

(b) V VELOCITY CONTOURS

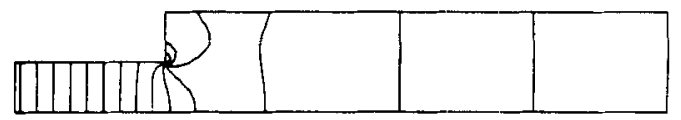

(c) PRESSURE CONTOURS

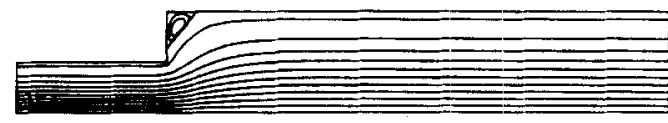

(d) STREAMLINES

Figure 5. Results at $R e=0$ : (a) $x$-velocity $u$; (b) $y$-velocity $v$; (c) pressure $p$; (d) streamlines

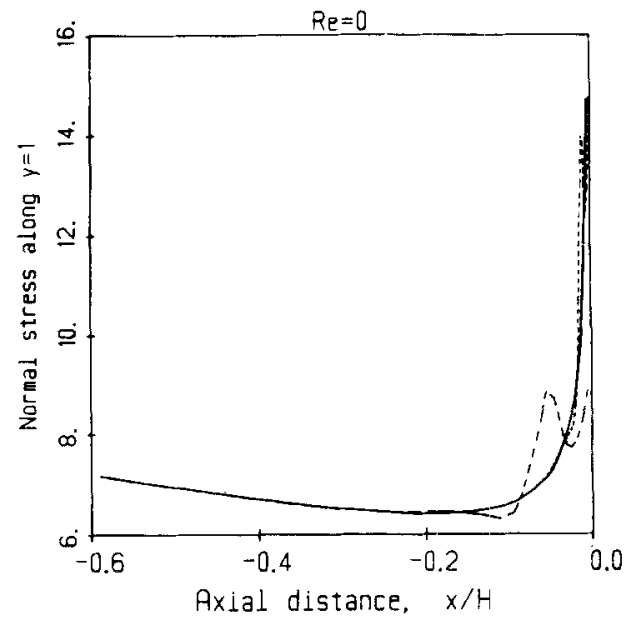

Figure 6. Normal stresses along the horizontal wall $(y=1)$ at $R e=0$ with OM2 (---), OM4 (- - ) and SM1 (- - ) 


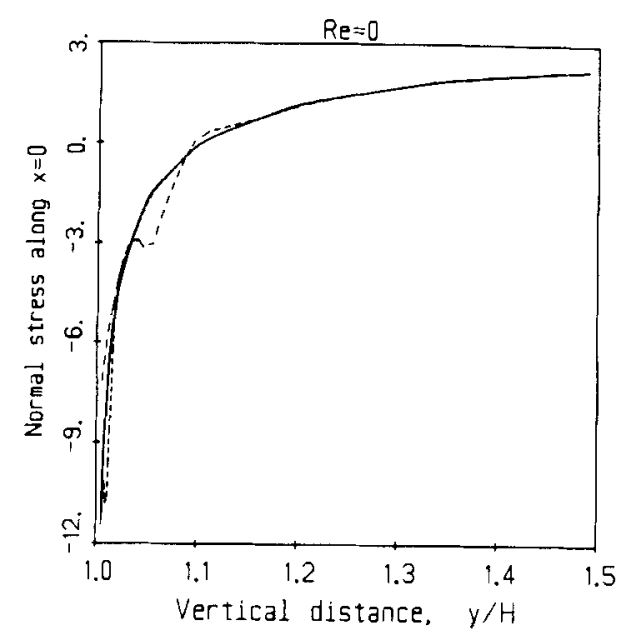

Figure 7. Normal stresses along the vertical wall $(x=0)$ at $R e=0$ with OM2 (---), OM4 (-..) and SM1 (-

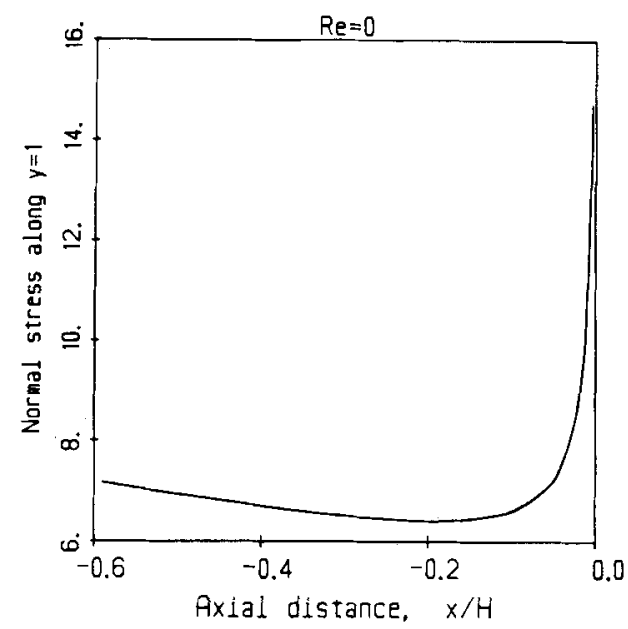

Figure 8. Normal stresses along the horizontal wall $(y=1)$ at $R e=0$ with SM1 and $n_{1}=0 \cdot 2(---), 0 \cdot 8(---)$ and 0.544( (all the curves essentially coincide)

smooth and become infinite at the singular point, as they should. Figures 6 and 7 suggest that the singular elements give more accurate results, with no oscillations for relatively coarse meshes.

One of the goals in this investigation was to study the sensitivity of the results to the powers $n_{i}$ of the basis functions. In Figure 8 we plot the normal stresses along the horizontal wall predicted with $n_{1}=0.544,0.2$ and 0.8 . We kept the values of the other powers constant. The results agree well, indicating that the normal stresses are rather insensitive to the powers $n_{i}$. Changing the value of the second exponent $n_{2}$ from 0.909 to 0.5 and 1.5 reaffirmed this conclusion. It seems that the removal of the pressure node from the singular point plays a more crucial role in improving the solution than the accuracy of $n_{i}$.

Next we applied singular finite elements at non-zero Reynolds numbers, $R e=0,1,10,50$ and 100. Again, the singular finite elements give the same solution far from the corner as the ordinary 
finite elements. The streamlines predicted with mesh SM1 are shown in Figure 9; the reattachment lengths are in good agreement with the results in Reference 28 . The line of separation on the vertical wall moves towards the corner as $R e$ is increased.

Once again, a more severe test is to compare the normal stress results. In Figures 10 and 11 the predicted normal stresses along the horizontal wall from meshes OM2, OM4 and SM1 have been plotted for $R e=10$ and 50 . The singular finite elements give more accurate results for relatively coarse meshes. Similar results were obtained for the normal stresses along the vertical wall $x=0$. All singular meshes give essentially the same results, indicating that smaller singular elements are

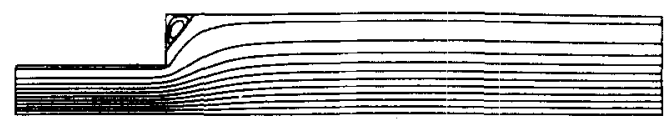

(a) $\quad \mathrm{Re}=0$

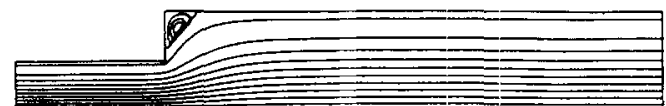

(b) $\mathrm{Re}=1$

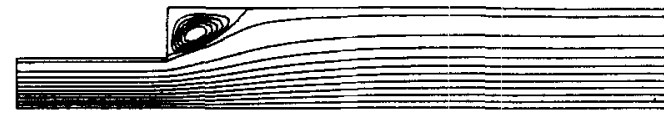

(c) $\mathrm{Re}=10$

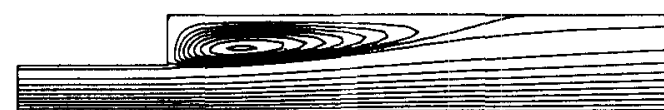

(d) $\mathrm{Re}=\mathbf{5 0}$

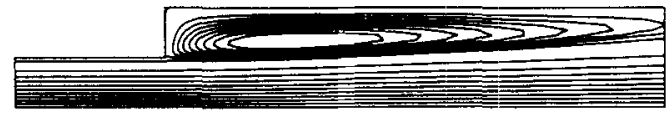

(e) $\mathrm{Re}=100$

Figure 9. Streamlines at diferent $\operatorname{Re}$ (with mesh SM1): (a) $R e=0$; (b) $\operatorname{Re}=1$; (c) $R e=10$; (d) $\operatorname{Re}=50$; (e) $\operatorname{Re}=100$. The maximum values of the streamfunction in the vortex are 1.0006, 1.0010,1.0124, 1.0412 and 1.0476 respectively

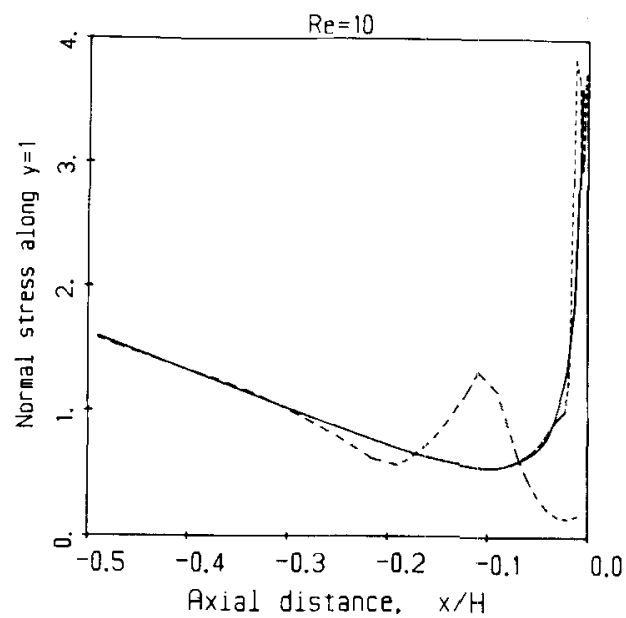

Figure 10. Normal stresses along the horizontal wall $(y=1)$ at $R e=10$ with OM2 (---), OM4 (-.-) and SM1 ( - 


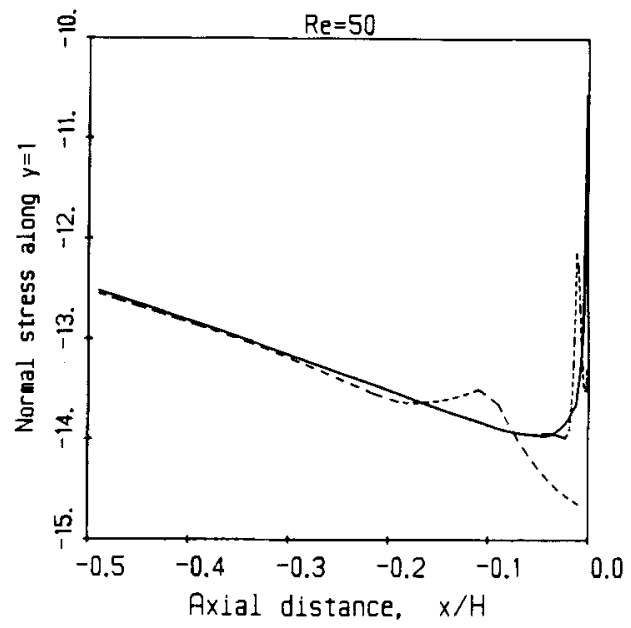

Figure 11. Normal stresses along the horizontal wall $(y=1)$ at $R e=50$ with OM2 (---), OM4 (-.-) and SM1 ( $\longrightarrow$ )

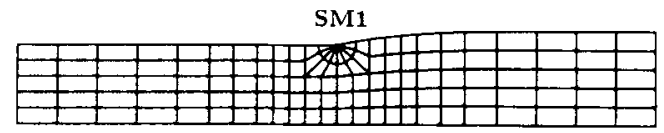

SM2

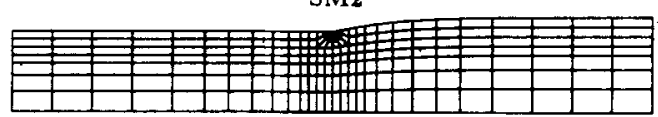

SM3

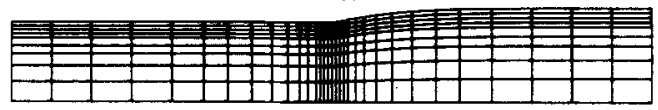

Figure 12. Singular meshes for the die-swell problem

not necessary for the non-zero Reynolds number flow. This, in conjunction with the previous observation that the normal stresses are rather insensitive to the powers $n_{i}$, gave us confidence to proceed to the solution of the die-swell problem using the SFEM.

\subsection{The die-swell problem}

For the die-swell computations we constructed three ordinary (OM1, OM2 and OM3) and three singular meshes (SM1, SM2 and SM3). The singular meshes, shown in Figure 12, were again obtained by modifying the corresponding ordinary meshes. Data about all meshes are given in Table III. The meshes extend up to four channel half-widths upstream and downstream. Again, as the Reynolds number increases we must increase the downstream length $L_{2}$. As we mentioned previously, full Newton iteration is used to solve this free surface problem. The free surface profile is computed simultaneously with the velocity and pressure fields, and the mesh is updated according to the position and shape of the free surface. A zero-order continuation is used for both parameters involved, $R e$ and $C a$, to proceed to higher $R e$ and lower $C a$. 
Table III. Data for die-swell meshes $\left(L_{2}=4\right)$

\begin{tabular}{lcccc}
\hline Mesh & $\begin{array}{c}\text { Number } \\
\text { of elements }\end{array}$ & $\begin{array}{c}\text { Number } \\
\text { of nodes }\end{array}$ & $\begin{array}{c}\text { Degrees } \\
\text { of freedom }\end{array}$ & $\begin{array}{c}\text { Size of } \\
\text { corner elements }\end{array}$ \\
\hline OM1 & 120 & 539 & 1253 & 0.20 \\
OM2 & 196 & 855 & 1971 & $0 \cdot 10$ \\
OM3 & 288 & 1235 & 2833 & 0.05 \\
SM1 & 128 & 597 & 1401 & $0 \cdot 24$ \\
SM2 & 204 & 913 & 2119 & 0.12 \\
SM3 & 296 & 1293 & 2981 & 0.06 \\
\hline
\end{tabular}

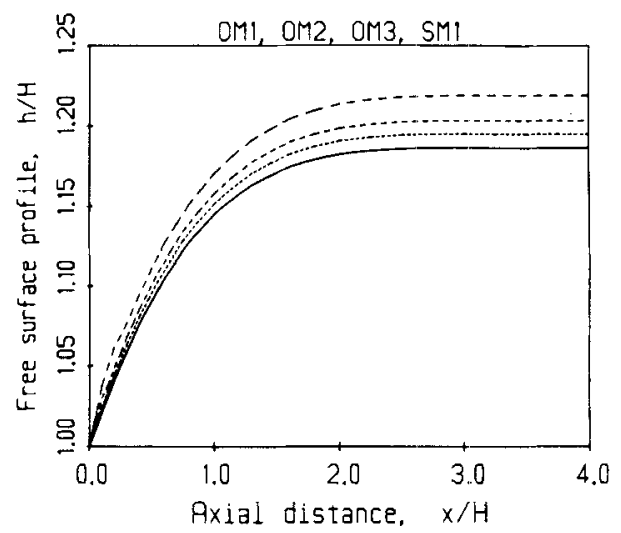

Figure 13. Computed free surface profiles at zero $R e$ and zero surface tension with OM1 (---), OM2 (---), OM3 (---) and SM1 $(\longrightarrow)$

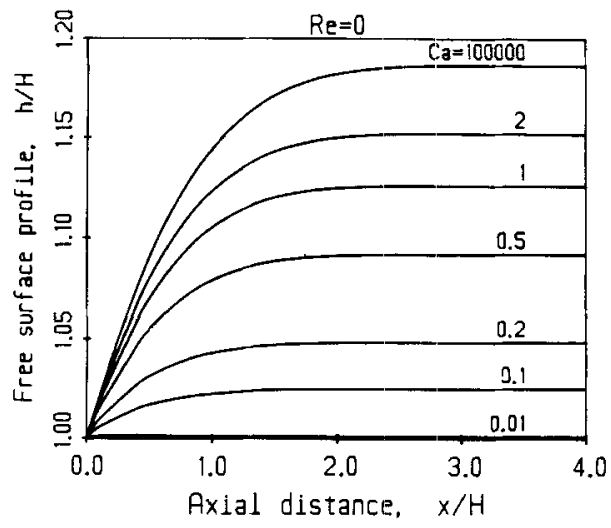

Figure 14. Free surface profiles at various $\mathrm{Ca}$ and zero $\mathrm{Re}$

The obvious choice for comparisons between ordinary and singular finite elements is the free surface profile. In Figure 13 we plot the predicted free surface profiles for all the ordinary meshes and SM1. All the singular meshes gave practically the same results and predicted the same dieswell ratio $(1 \cdot 186)$. As we see in Figure 13, the ordinary elements converge slowly to the solution 
obtained by a relatively coarse singular mesh. The singular elements speed up the convergence of the free surface considerably.

Results have been obtained for various $R e$ and $C a$. The SFEM solution in the neighbourhood of the singularity proved to be insensitive to variations of the powers $n_{i}$, supporting the use of the same singular elements at any apparent angle of separation or at different capillary numbers. The free surface profiles for various capillary numbers at $R e=0$, obtained with mesh SM1, are plotted in Figure 14.

In closing, we should note that the SFEM is not free of shortcomings or limitations. The radial form of the singularity must be known (to a certain accuracy) and additional programming is required. The method also leads to stiffness matrices with bigger semibandwidths or frontwidths than those from ordinary element meshes. Nevertheless, this is the price one must pay for the improved accuracy and the faster convergence.

\section{CONCLUSIONS}

Singular finite elements have been used to solve the $2: 1$ sudden-expansion and the die-swell problems at various Reynolds numbers ranging from zero to 100 . The singular elements surround the singular point and have no pressure node there. The corresponding basis functions embody the form of the singularity for each case. The elements used for creeping flow were used to solve the problem for non-zero Reynolds numbers because the form of the local solution remains unchanged very close to the singular point. The method gives more accurate results than those from more refined ordinary finite element meshes; in addition, no oscillations are observed. The normal stress results were found to be rather insensitive to the singularity powers used in the construction of the singular basis functions.

For the die-swell problem, the singular elements developed for the stick-slip problem have been used. The singular elements speed up the convergence of the free surface considerably.

\section{ACKNOWLEDGEMENTS}

This work was partially supported by The Ocean Engineering Division of the Office of Naval Research contract N00014-87-0509, and the National Science Foundation contract DMC8716766.

\section{REFERENCES}

1. R. A. Brown, R. C. Armstrong, A. N. Beris and P.-W. Yeh, 'Galerkin finite element analysis of complex viscoelastic flows', Comput. Methods Appl. Mech. Eng., 58, 201 (1986).

2. J. M. Marchal and M. J. Crochet, 'A new mixed finite element for calculating viscoelastic flow', J. Non-Newtonian Fluid Mech., 26, 77 (1987).

3. W. J. Silliman and L. E. Scriven, 'Separating flow near a static contact line. Slip at a wall and shape of a free surface', J. Comput. Phys., 34, 287 (1980).

4. M. R. Apelian, R. C. Armstrong and R. A. Brown, 'Impact of the constitutive equation and singularity on the calculation of stick-slip flow: the modified upper-convected Maxwell model (MUCM)', J. Non-Newtonian Fluid Mech., 27, 299 (1988).

5. J. R. Rosenberg and R. Keunings, 'Further results on the flow of a Maxwell fluid through an abrupt contraction', J. Non-Newtonian Fluid Mech., 29, 295 (1988).

6. H. Gallagher, 'Finite element analysis for crack tip problems', in Proc. Symp. on Finite Element Method, Science Press, Beijing 1982, p. 102.

7. G. J. Fix, 'Singular finite element methods', in D. L. Dwoyer, M. Y. Hussaini, and R. G. Voight (eds), Finite Elements. Theory and Application, Springer-Verlag, New York, 1988, Chap. 3.

8. H. Holstein and D. J. Paddon, 'A singular finite difference treatment of re-entrant corner flow', J. Non-Newtonian Fluid Mech., 8, 81 (1981).

9. D. B. Ingham and M. A. Kelmanson, Boundary Integral Equation Analyses of Singular, Potential, and Biharmonic Problems, Springer-Verlag, Berlin, 1984, p. 21. 
10. G. C. Georgiou, L. G. Olson, W. W. Schultz and S. Sagan, 'A Singular finite element for Stokes flow: the stick-slip problem', Int. j. numer. methods fluids, 9, 1353-1367 (1989).

11. G. C. Georgiou, L. G. Olson and W. W. Schultz, 'Two finite element methods for singularities in Stokes flow: the stick-slip problem', in T. J. Chung and G. R. Karr (eds), Finite Element Analysis in Fluids, UAH Press, Huntsville, Alabama, 1989, p. 992.

12. D. H. Michael, 'The separation of a viscous liquid at a straight edge', Mathematika, 5, 82 (1958).

13. H. K. Moffatt, 'Viscous and resistive eddies near a sharp corner', J. Fluid Mech., 18, 1 (1964).

14. L. D. Sturges, 'Die swell: the separation of the free surface', J. Non-Newtonian Fluid Mech., 6, 155 (1979).

15. G. G. Lipscomb, R. Keunings and M. M. Denn, 'Implications of boundary singularities in complex geometries', J. Non-Newtonian Fluid Mech., 24, 85 (1987).

16. P. Henriksen and O. Hassager, 'Corner flow of power law fluids', 10th Int. Congr. on Rheology, Sydney, 1988.

17. A. R. Davies, 'Re-entrant corner singularities in non-Newtonian flow. Part I: Theory', J. Non-Newtonian Fluid Mech., 29, 269 (1988).

18. S. A. White, A. D. Gotsis and D. G. Baird, 'Review of the entry flow problem: experimental and numerical', J. NonNewtonian Fluid Mech., 24, 121 (1987).

19. B. J. Omodei, 'Computer solutions of a plane Newtonian jet with surface tension', Comput. Fluids, 7,79 (1979).

20. R. E. Nickell, R. I. Tanner and B. Caswell, 'The solution of viscous incompressible jet and free-surface flows using finite element methods', J. Fluid Mech., 65, 189 (1974).

21. K. J. Ruschak, 'A method for incorporating free boundaries with surface tension in finite element fluid-flow simulators', Int. j. numer. methods eng., 15, 639 (1980).

22. G. C. Georgiou, T. C. Papanastasiou and J. O. Wilkes, 'Laminar Newtonian jets at high Reynolds and high surface tension', AIChE J., 34, 1559 (1988).

23. S. Richardson, 'A 'stick-slip' problem related to the motion of a free jet at low Reynolds numbers', Proc. Camb. Phil. Soc., 67, $477(1970)$.

24. W. W. Schultz and C. Gervasio, 'An analysis of the singularity in the die swell problem', 10th Int. Congr. on Rheology, Sydney, 1988.

25. T. J. R. Hughes and J. E. Akin, 'Techniques for developing "special" finite element shape functions with particular reference to singularities', Int. j. numer. methods eng., 15, 733 (1980).

26. P. Hood, 'Frontal solution program for unsymmetric matrices', Int. j. numer. methods eng., 10, 379 (1976).

27. J. S. Solecki and J. L. Swedlow, 'On quadrature and singular finite elements', Int. j. numer. methods eng., 20, 395 (1984).

28. P. S. Scott, F. A. Mirza and J. Vlachopoulos, 'A finite element analysis of laminar flows through planar and axisymmetric abrupt expansions', Comput. Fluids, 14, 423 (1986).

29. D. F. Fletcher, S. J. Maskell and M. A. Patrick, 'Heat and mass transfer computations for laminar flow in an axisymmetric sudden expansion', Comput. Fluids, 13, 207 (1985).

30. P. M. Gresho and R. L. Lee, 'Don't suppress the wiggles - they're telling you something', Comput. Fluids, 9, 223 (1981). 